\title{
“Green Building” - Modelling renewable building energy systems and electric mobility concepts using Modelica
}

\author{
René Unger ${ }^{1}$, Torsten Schwan ${ }^{2}$, B. Mikoleit ${ }^{1}$, Bernard Bäker ${ }^{2}$, Christian Kehrer ${ }^{3}$, Tobias Rodemann ${ }^{4}$ \\ ${ }^{1}$ EA EnergieArchitektur GmbH, Dresden, Germany \\ ${ }^{2}$ Institute of Automotive Technologies Dresden - IAD, Dresden University of Technology \\ Dresden, Germany \\ ${ }^{3}$ ITI GmbH, Dresden, Germany \\ ${ }^{4}$ Honda Research Institute Europe, Offenbach/Main, Germany \\ rene.unger@ea-gmbh.de,schwan@iad.tu-dresden.de,kehrer@iti.de \\ Tobias.Rodemann@honda-ri.de
}

\begin{abstract}
For most people, a comfortable living and mobility are basic needs. With the rising individual demand for energy as well as the diminishing fossil energy resources, new optimized concepts for energy supply and usage are required. To address these challenges, renewable energy sources, decentralized storage, and electric mobility concepts are matters of rapidly growing importance.

Future building energy systems have to successfully integrate user demands, local renewable energy, storage systems and charging infrastructure, a task requiring extensive scrutinizing.

Typical questions to the engineer are to compare different system layouts with respect to sustainability, cost, and robustness, or to identify the right levers in an energy system to optimize components and control algorithms.

This paper describes an approach to solve such questions using simulations with the non-causal language Modelica. Modeling paradigms and examples are shown. Special emphasize is given to the "Green Building" library and its components, bringing major building energy systems and electric vehicles to the same platform.
\end{abstract}

Keywords: Renewable energy; Building; eMobility

\section{Introduction}

Increasing the use of renewable energy for almost all aspects of people's life is one of the major topics of this decade. Energy storage, smarter energy consumption and interaction of energy grid components, on global scale as well as locally, are tasks to be solved by the engineer.

Ecological footprint, detail efficiency as well as usage comfort are matters becoming more important. To fulfill these aspects, various components like photovoltaic or storage tanks, even weather forecast, need to work together to satisfy the users' demands in a renewable and reliable way.

In addition to the technical aspects of automation and networking systems, the functionality of this component interaction needs to be clarified. Heat, electricity and mobility used to be separated aspects in life. With the use of renewables these are increasingly correlated. For example a combination of micro-wind-turbines, photovoltaics, solar heat and heat pumps could be used in a specific building. Another possible solution would be a combined heat and power unit (CHP) heating the house and charging the electric vehicle. The solution may also vary depending on the available monetary budget.

Renewable energy is limited in availability. The peak PV-power is at noon while peak consumption is often in the morning or in the evening. The need for energy storage or at least time shifting of consumption arises. Even user behavior is important in such a system. 

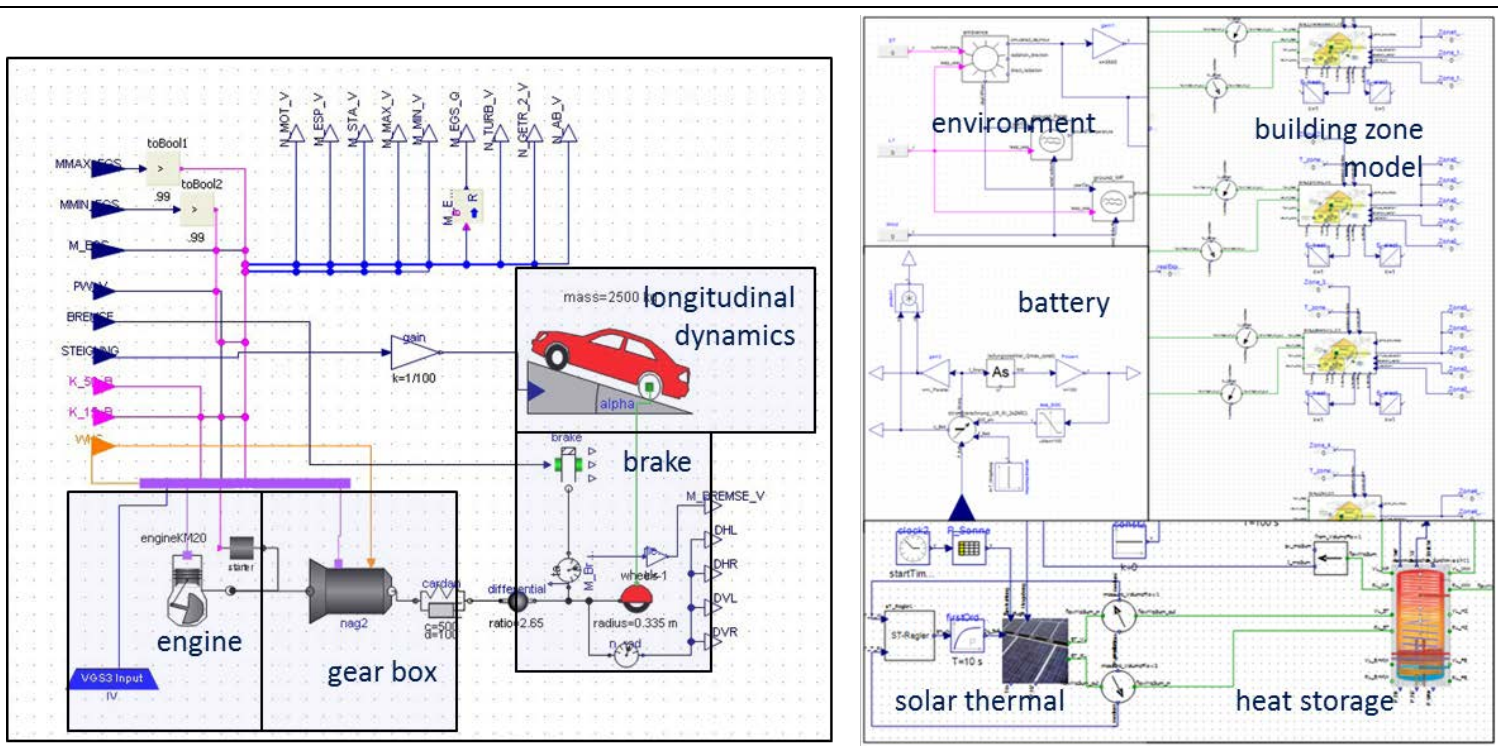

Fig. 1: Modelica-based simulation models for vehicles (left) and buildings (right) [1, 5]

Hence, to find a suitable energy system configuration for a specific scenario has become an extensive engineering task. Therefore, new supporting tools and methods covering the whole system are needed.

\section{Simulation Tools}

Today's available tools can be categorized into different groups. First, there are special component layout programs like for example PVSol for photovoltaics.

A second group of tools uses FEM and CFD. These make it possible to simulate heat and radiation input to complex rooms and buildings and to calculate the resulting temperature fields, air flow, etc. EnergyPlus, ANSYS and Ecotect are examples for these powerful tools.

A third group addresses systems simulation. HVAC, even photovoltaics and wind are integrated into one block oriented system model. The underlying physics are often represented as equivalent networks while control algorithms are represented in a signal oriented way or are programmed in a procedural language. A typical tool-chain would contain TRNSYS and Matlab Simulink. These toolchains are extraordinary powerful. Yet some important effects like the nondeterministic behavior of humans, electric mobility, dynamic costs, battery aging and probability-based energy management systems have been difficult to implement.

Modelica, as a non-causal, non-proprietary and cross-domain modeling language with Tools like SimulationX excel in these requirements. Some $\mathrm{Li}$ braries like Modelica "Buildings" or "Human Comfort" contain models for building energy and com- bined control system simulations [8]. Yet, with these libraries, it is still a huge effort to model a complete building-vehicle-user energy system.

In such a holistic simulation, systems of fast dynamics (1ms-1s) like vehicle physics or battery control have to work alongside with systems of low dynamics (1min-1day) for a long simulation time span (days to years).

Additionally the models need to be complex enough to test control algorithms but not too complex for a fast simulation speed as well as safe and easy to configure. A systems engineer as addressee of the simulation is specialized in component interaction, not in heat pump specifics, vehicle batteries and detailed building thermodynamics. For special cases where detail is needed, simulator coupling is an option.

To fulfill these requirements, an approach widely used in the automotive industry was adapted to the field of building energy system modeling and realized in the Green Building Simulation Library.

\section{Modelica Green Building Library - modeling paradigms}

The Green Building Library was created using the cross-domain equation based concept of the Modelica language. The aim was to create a set of physical and functional models with similar granularity and handling. This way a complete renewable energy system can be represented (Fig. 2), including:

- sources like photovoltaics, windmills, solarthermal collectors, heat pumps or CHPs,

- storage tanks, batteries and grid,

- consumers, user behavior, weather as well as

- charging stations and electric vehicles. 


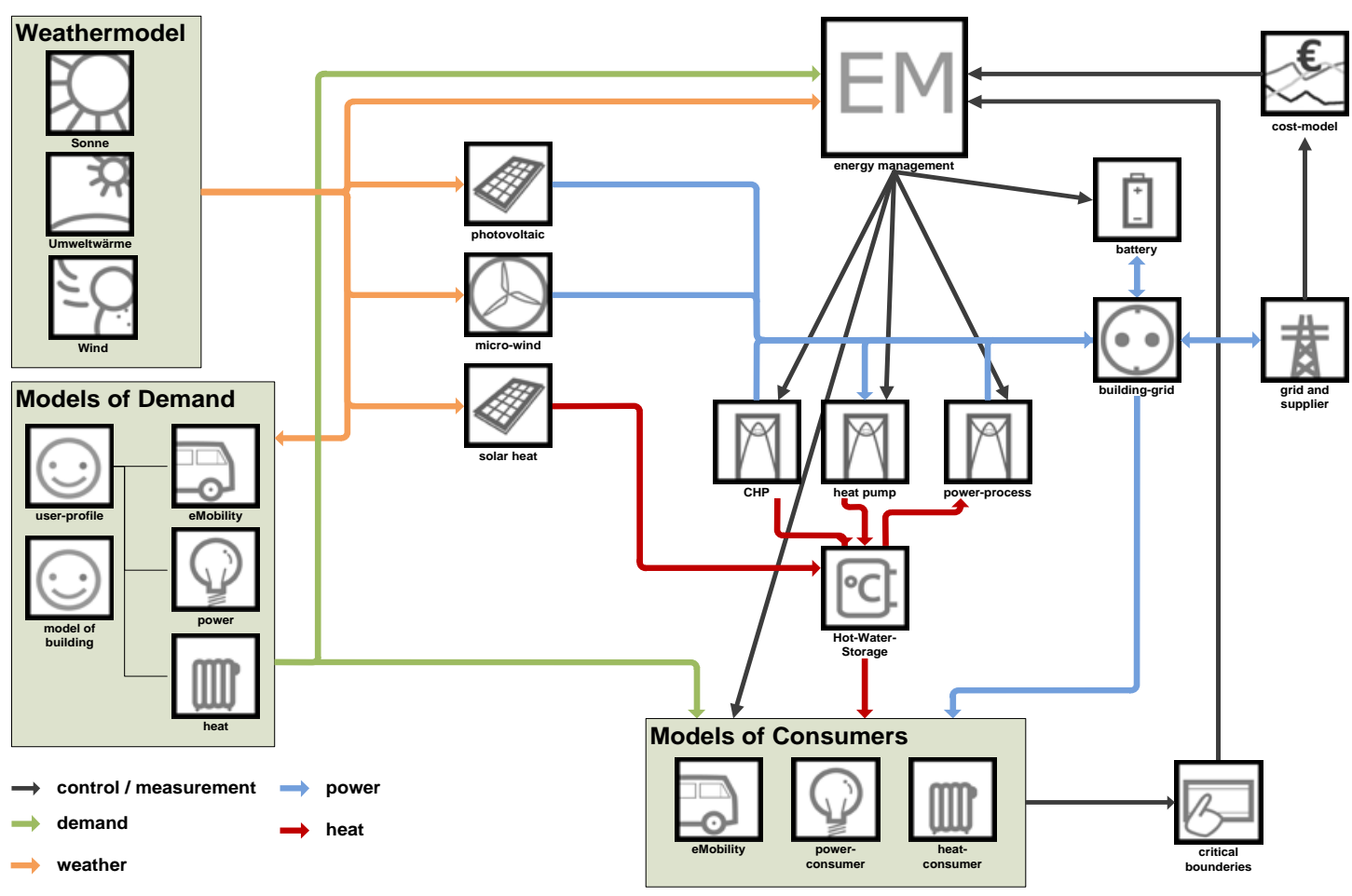

Fig. 2: Component models integrated in Modelica-library [2]

The granularity and complexity (fig. 1) of each element is comparable to the approach used in the automotive industry (e.g. partial models for engines, gearboxes and longitudinal dynamics of vehicles).

Special emphasis was placed on the input parameter set of each component and intuitive modeling. For better usability, all parameters are similar to those found in typical component data sheets or standardized reports (i.e. EnEV [10]).

Another requirement to the chosen approach is, that all the relevant characteristics needed for the comparison of different building energy systems, shall be calculated within one simulation environment, if possible. Therefore, all library components have been modeled as compatible differentialalgebraic equation systems (DAE) including physical behavior, control algorithms and external interrupt connectors for energy management systems. Furthermore, optional functions to calculate investment and operating costs of each component can be used (fig. 3). Currently, however, these calculations are performed in external post processing routines.

Another important requirement is a high simulation speed while maintaining the highest possible time resolution. For advanced comparisons, a year needs to be simulated with a minimum step size of 1 minute; this is at least half a million steps for one simulation run. This is longer than the usual 15min $1 \mathrm{~h}$ time steps of thermal simulations. To achieve the needed model reduction fast internal processes were simplified or replaced with functional descriptions, each as internal DAE systems.

A major factor for the simplification was the influence on outer processes and component interaction. A typical example is the windmill power electronics. Instead of simulated MOSFETs and capacitors, a phenomenological model containing characteristic curves for conversion factors, efficiency and voltage limiters represents the electronics.

In other models, where such simplification is no option (error margin, numeric stability), precalculation is used. Complex driving cycles are one example for fast internal time constants. Heat pump characteristics based on source and heating medium temperatures are another example, where the solver would need to calculate partial differentials.

Emphasize is given to an exact representation on effects which are relevant for the energetic behavior of a component (e.g. heat absorption and dissipation of a heat storage depending on the temperature spread). Other equations describing less relevant effects, like volume flow within heat storages, are neglected. This constant granularity is a usability advantage over usage of models from different sources.

Influences like weather are integrated using an ambience block, which reads either external databases or location provided with the library as internal blocks (fig. 3).

Most of the library components require special control algorithms for the internal regulation of the 


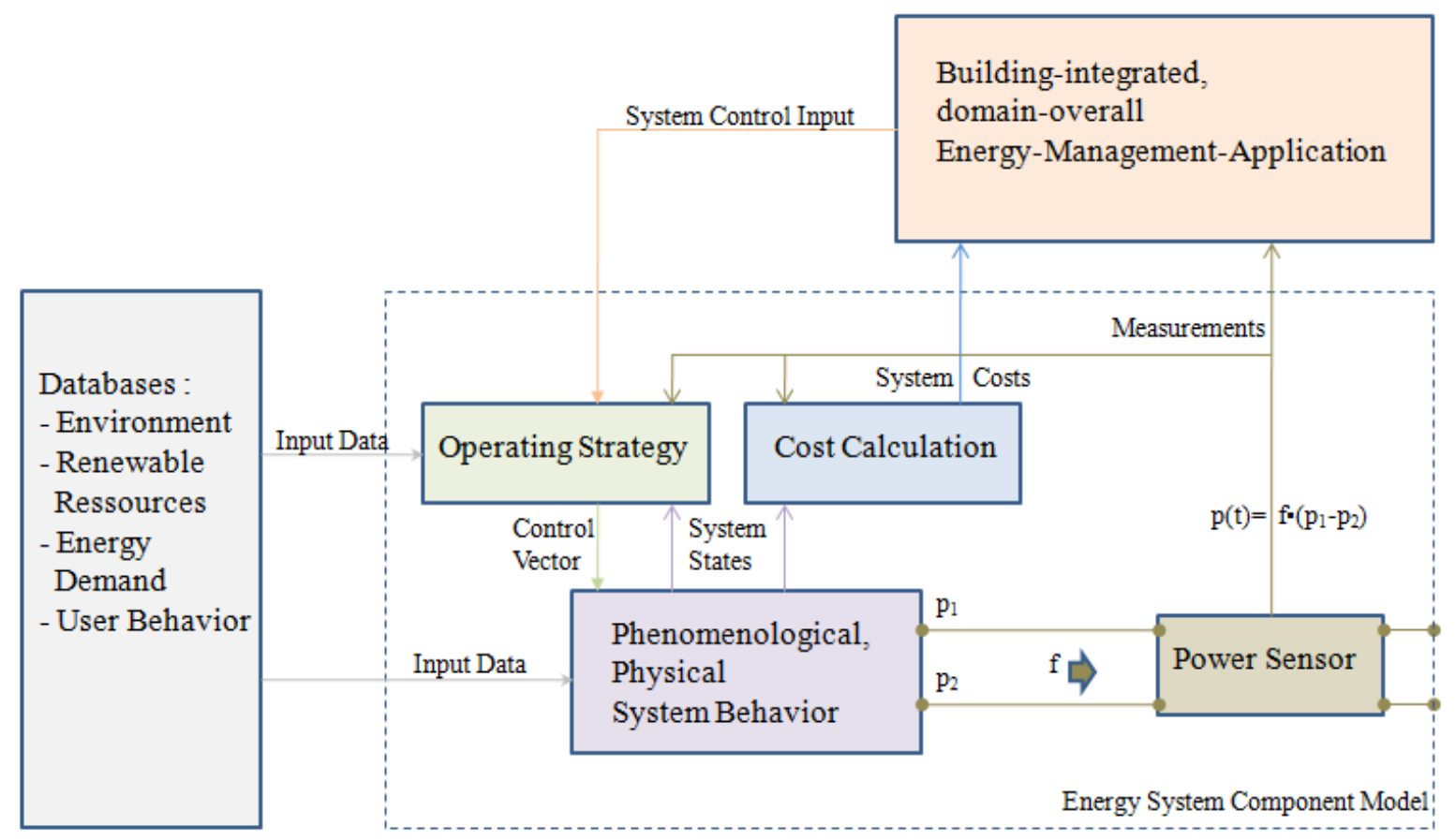

Fig. 3: Generalized component layout [1]

system states (e.g. de-icing processes of air heat pumps). These control strategies are integrated as blocks in the general component layout to simulate the typical behavior of the components. The user, to test new operating strategies like a combined heat/power-led CHP usage depending on vehicle dock and weather forecast, can replace the control block. Another option is the use of controller inputs for a superimposed energy management (fig. 3).

Within a domain, the components are connected with specific connector elements, which are derived from "real-world"-connections like pipes for heating systems and cables for electrical systems. This way, the real energy exchange is simulated and can be observed during and after each simulation run [1].

$$
\begin{aligned}
& e_{\text {therm }}(t)=\int \rho_{\text {med }} \cdot c_{p_{\text {med }}} \cdot \dot{V}_{\text {med }} \cdot \Delta T_{\text {med }} d t \\
& e_{e l}(t)=\int U_{\text {eff }} \cdot I_{\text {eff }} \cdot \cos \varphi \cdot n_{\text {phase }} d t
\end{aligned}
$$

Both consistent equations (1) and (2) describe the interchanged energy between connected components. Therefore, both equations consist of domain-specific flow (volume flow and current) and potential (temperature spread and voltage) states as well as further special constant values.

\section{Coupling of Building and Vehicle}

As explained before, the main challenge in coupling building and vehicle is the difference in the major time constants, which would lead to a small minimum simulation step size.
The thermal energy flow in buildings mainly depends on low system dynamics caused by inner masses, slow external temperature changes, etc. Electrical demand changes faster but it still is in the order of minutes at the building's lateral.

In contrast to that, vehicle-specific time constants are much lower, in the order of milliseconds to seconds and they are vital for an adequate simulation of the energetic behavior (fuel, electrical energy).

There are proven frameworks to dynamically simulate the energetic behavior of vehicles with alternative drive trains [see for example 9]. These contain approaches to simulate the driver's behavior and the operating strategy defining the vehicle operation mode (EV mode, ICE mode) as well as the detailed dynamic behavior of each vehicle component (e.g. gear boxes, ICE, EM etc.).

Unfortunately, such approaches are too complex for implementation in the combined simulation of buildings and vehicles. Because of the differing system dynamics, direct coupling of building and vehicle creates very stiff DAE systems. Hence, either the simulation time becomes non-acceptable or the results deteriorate in accuracy and numerical stability.

To solve this we looked at the vehicle from the building point of view. This way the vehicle is a component, which is either docked at home or executing a certain driving cycle. If it is available and connected to a charging station (battery electric or plug-in hybrid) then the vehicle is a consumer or an intelligent battery. The important information is the energy or fuel needed for a specific cycle, vehicle availability and if the vehicle is connected, the power 


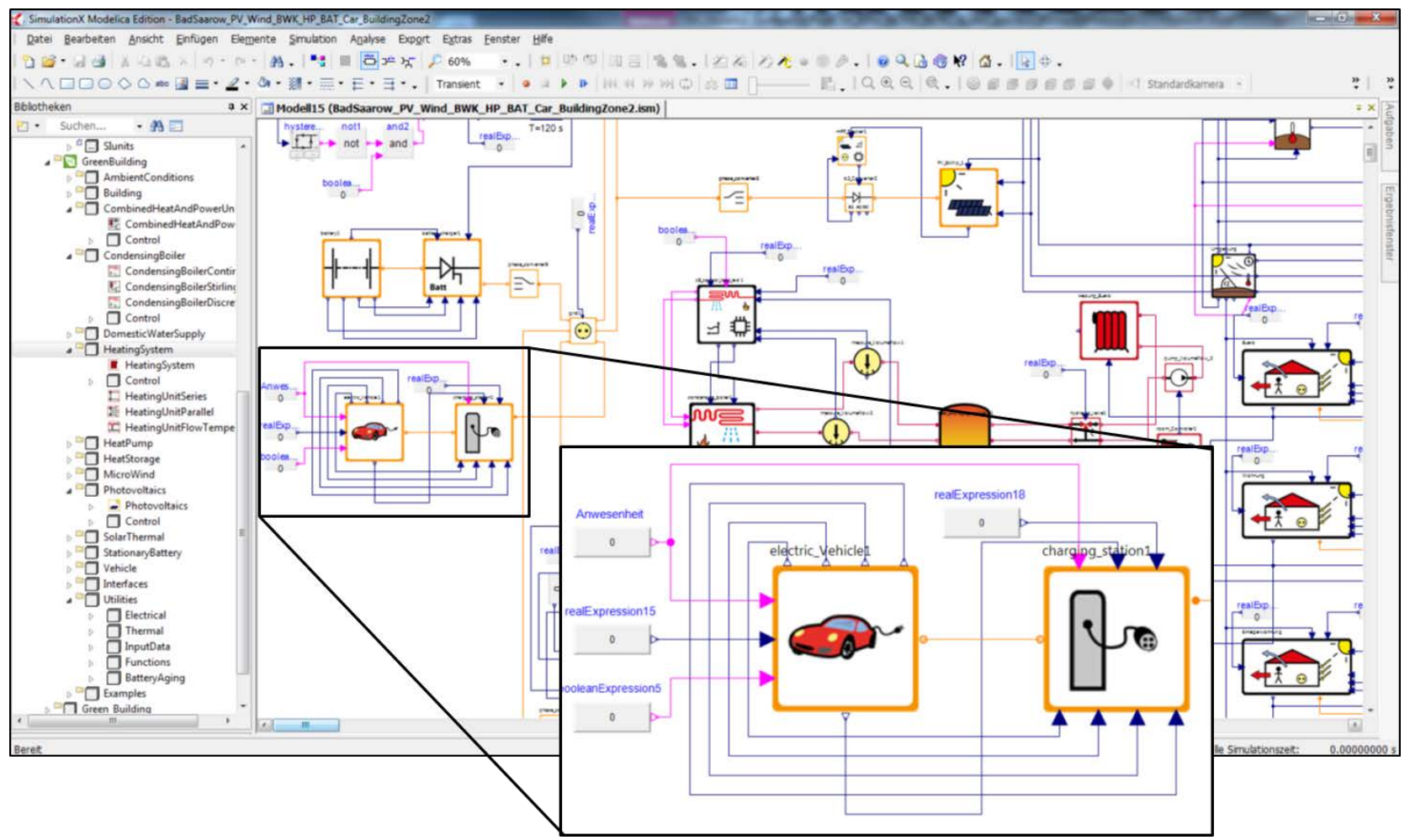

Fig. 4: Vehicle and charging station as a part of a complete energy system modeled in SimulationX

exchange and control algorithms. The energy demand during the driving cycle does not directly interact with the building. Therefore, this can be represented by pre-calculated driving cycles, user models and the simplification of the vehicle to a battery model.

This way in the building simulation, the vehicle is considered as only one component of the building energy system (fig. 4) with focuses on charging and grid-support. In a preprocessing operation, the driving cycles for different vehicle internals (ICE, BEV, PHEV, etc.) are simulated with high accuracy. This includes detailed longitudinal dynamics, architecture (serial, parallel etc.), power train components characteristics (e.g. battery size) and selected operating strategy (e.g. deplete and sustain) [see 9 et al.]. The main results are mean speed, mean power and fuel or energy consumption for use within the building energy simulation.

The vehicle presence can be either derived as a transient simulation variable from the driving time in the preprocessing or created as synthetic user behavior for the presence at the charging station.

Using that approach allows a high accuracy of the fuel and electrical power demand of the vehicle during a time period with an adequate simulation speed. Furthermore, the feedback of the charging strategy and the power supply to the vehicle on the building energy system can be analyzed. Influences of superior energy management systems on the electrical energy supply to the vehicle and the amount of renewable energy used for driving the vehicle are analyzable as well.

\section{Exemplary simulation results}

An example, showing capabilities and power of the presented "Green Building"-library is a semidetached house in Germany. One simulation represents a conventional energy system and ICE vehicle. A second simulation shows a complex renewable configuration.

The conventional and the renewable configuration are then assessed regarding:

- primary energy balance,

- carbon dioxide emission balance and

- renewable fraction of traction energy

For these assessments of annual balances, a statistical method was used. Analogue to VDI 4655, twelve reference days were defined (i.e. sunny winterday, weekend). Each reference day was simulated. The results were superimposed with the weather statistics of the last 10 years. 


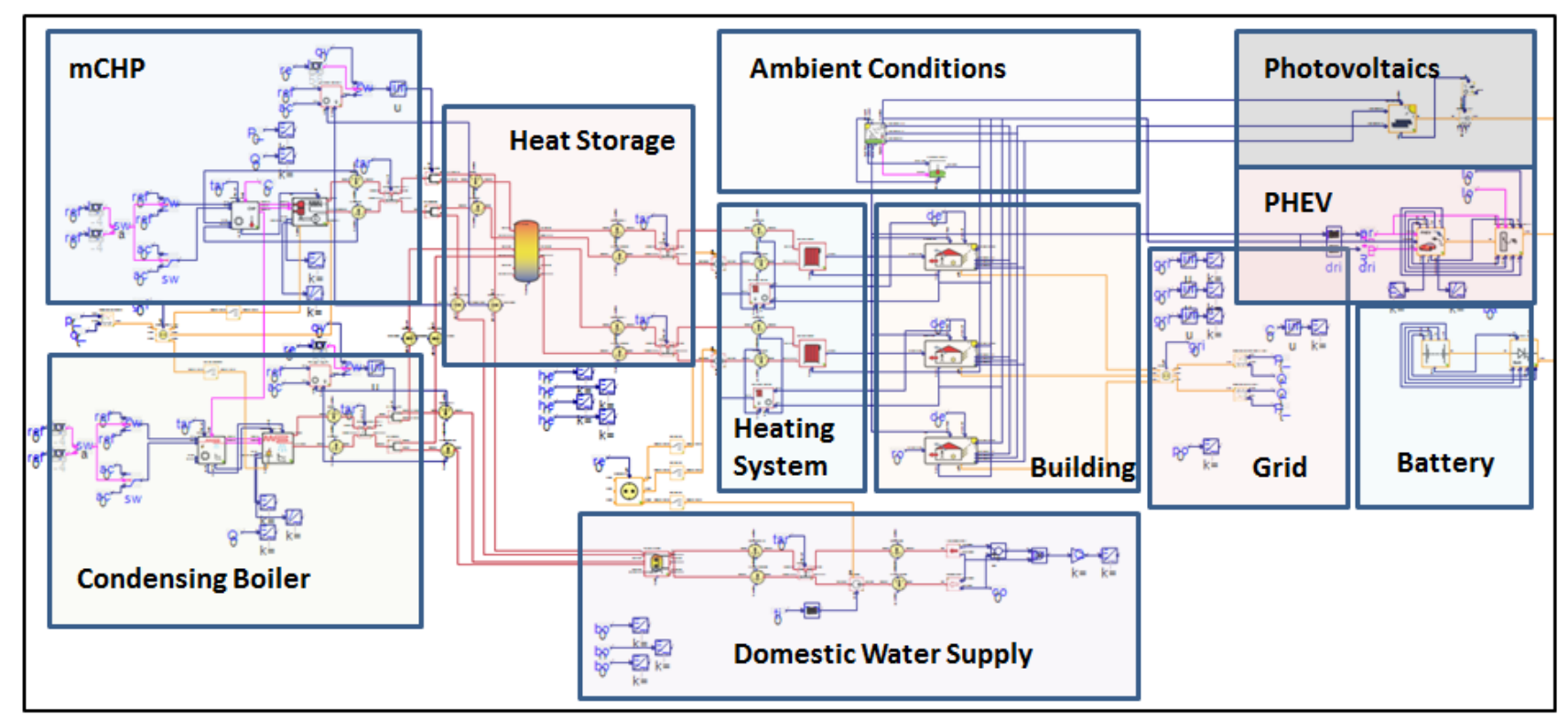

Fig. 5: Renewable energy system model

The renewable energy system consists of (fig. 5):

- small combined heat and power unit

- gas-fired condensing boiler

- heat storage

- domestic water boiler

- stationary battery

- photovoltaic system

- plug-in-hybrid-electric-vehicle

In opposite to that, the conventional energy system only contains three components (fig. 6):

- gas-fired condensing boiler

- domestic water boiler

- compact car (ICE)

Both systems were simulated in combination with a 3-zonal building model with floor heating system. Component parameters (e.g. battery size) and system control algorithms were adapted to the requirements of the exemplary building scenario. However, parameter variations (e.g. variation of battery size) could easily be done due to the flexibility of the library components.

Although, there are many other analyzable criteria, for renewable energy systems, the ecological footprint is one of the most important bases of the decision-making. It is possible to evaluate this footprint by using primary energy factors. These factors bias different forms of energy (e.g. electricity, fuel, natural gas) by describing how much primary energy (e.g. coal-equivalent) is required for their provision.

Fig. 7 shows the primary energy balance of the analyzed system variants. The renewable system has a better primary energy balance and ecological footprint than the conventional system since a big share of the electrical energy is generated by CHP and photovoltaics.

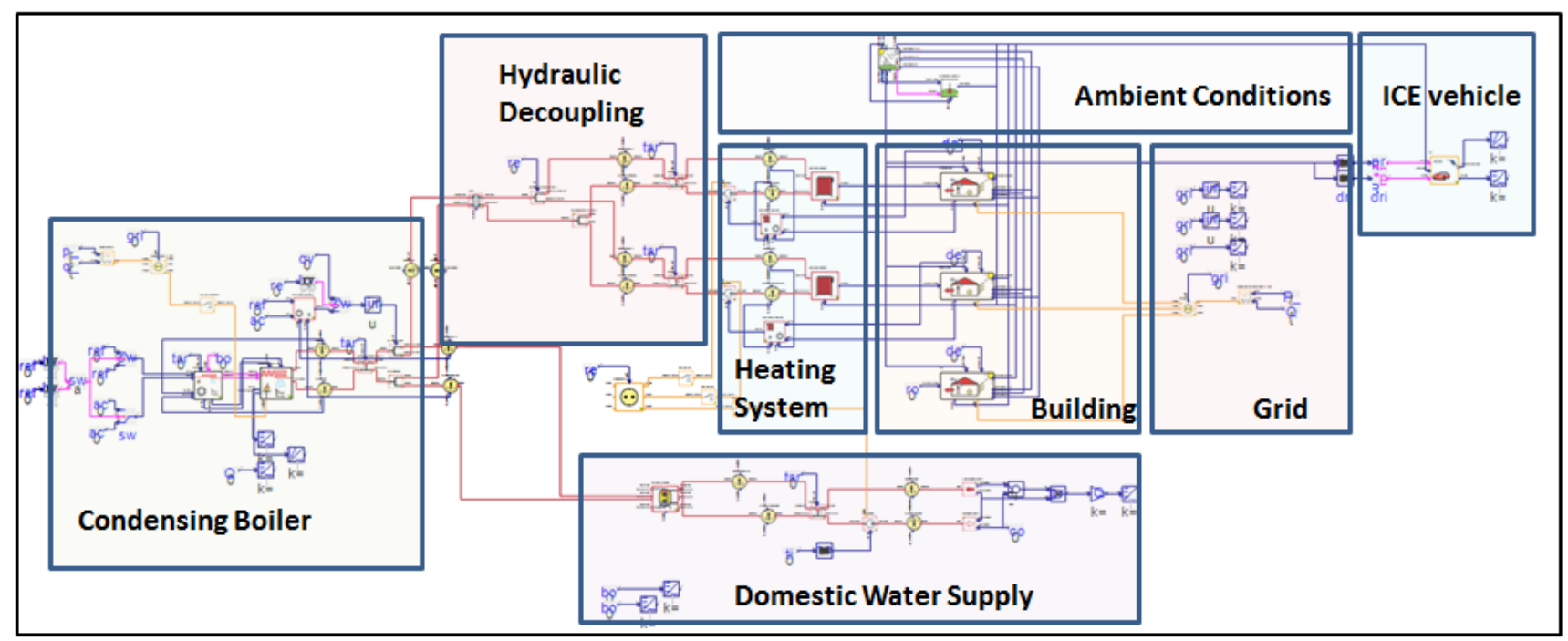

Fig. 6: Conventional energy system model 


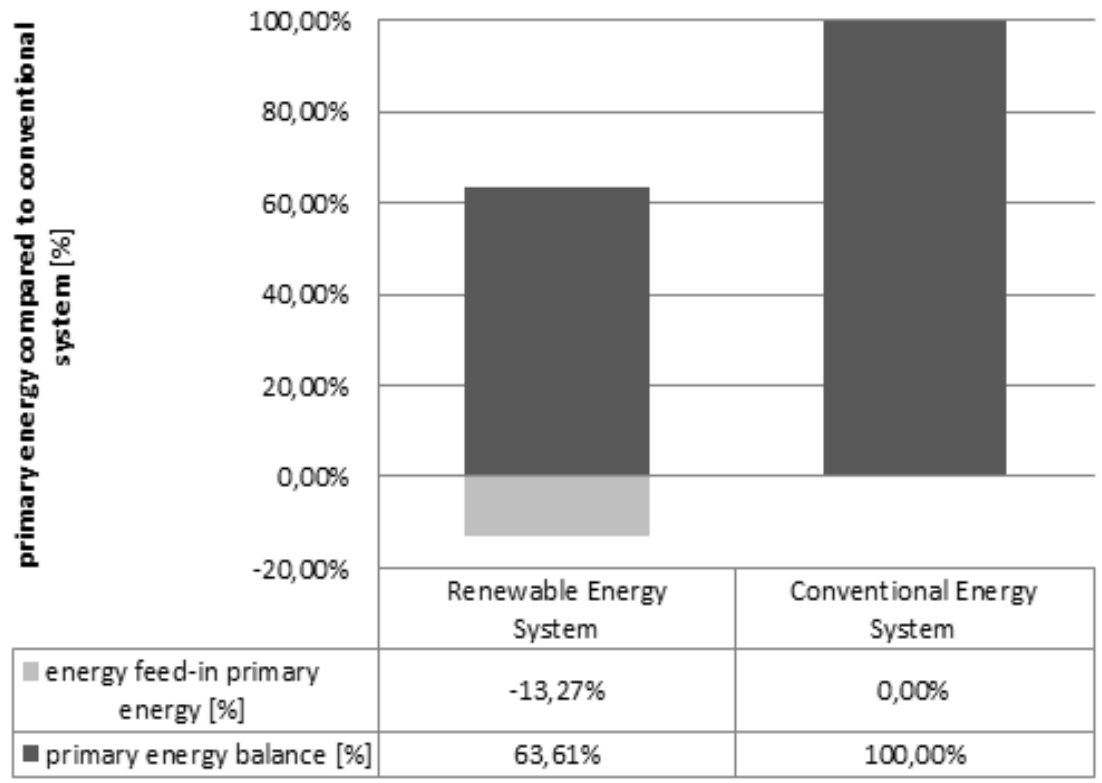

Fig. 7: Comparison of relative primary energy balance

Another important criterion for evaluation of different building energy systems and connected vehicles with (partly) electrified power train is the annual carbon dioxide emission balance. In this process all emissions of vehicles, heating system and electrical energy consumption are analyzed.

Fig. 8 shows that the annual $\mathrm{CO}_{2}$ emission and electrical energy consumption of the renewable system with PHEV are much lower than the ones of conventional system. The main reason is the electricity output of photovoltaics and CHP. This helps to maintain a high renewable mileage of the PHEV in a typical commuting situation with $30 \mathrm{~km}$ of daily driving. With the regarded vehicle, this cycle could be covered almost completely on battery. The only exceptions were some winter days where the combustion engine was needed.

Compared to the conventional system, the $\mathrm{CO}_{2}$ emission for heating in the renewable system is higher. This is caused by electricity conversion and by the marginally lower thermal efficiency of the CHP (92\% of CHP compared to 98\% of condensing

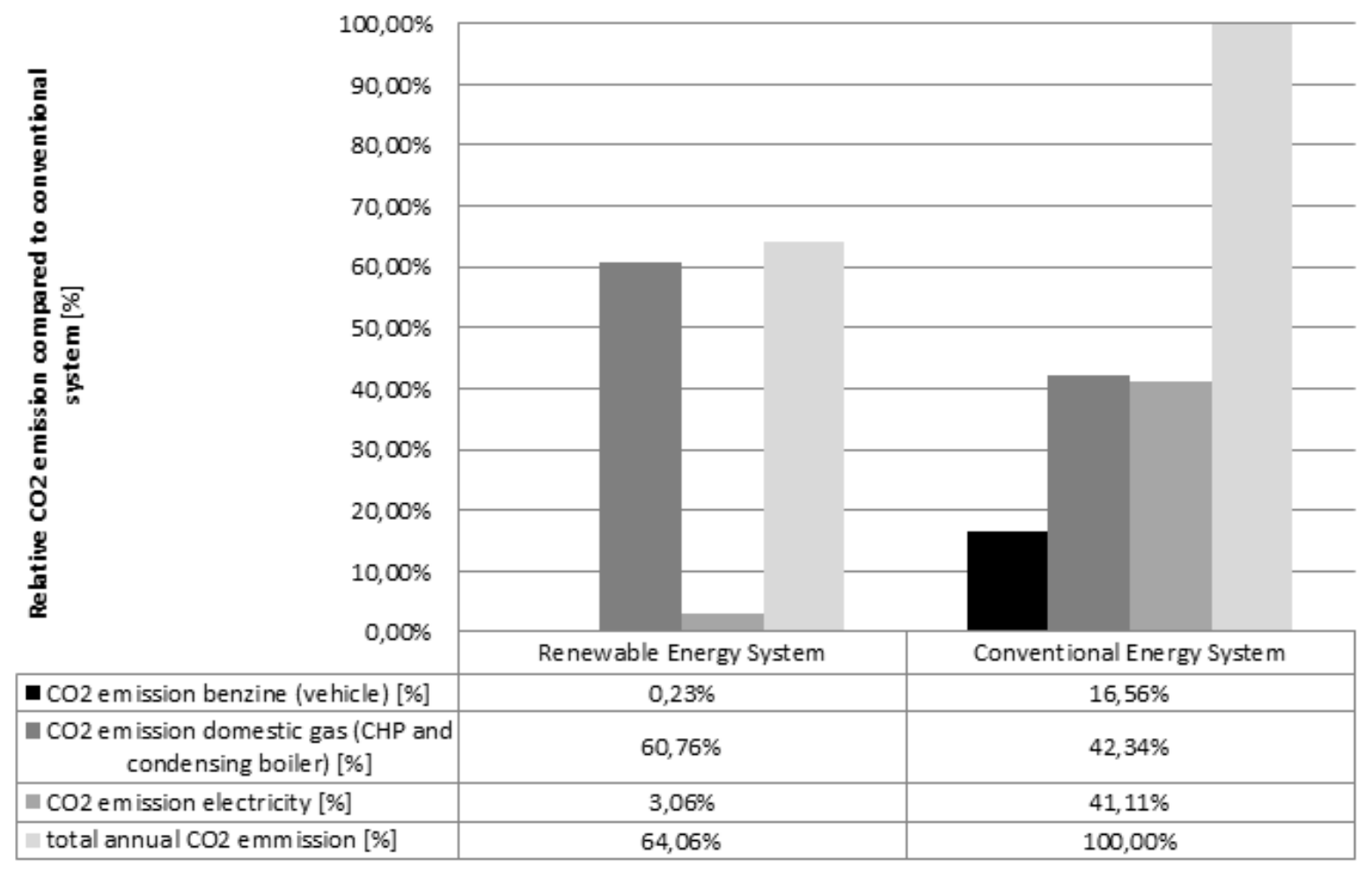

Fig. 8: Comparison of relative primary energy balance 
“Green Building” - Modelling renewable building energy systems and electric mobility ...

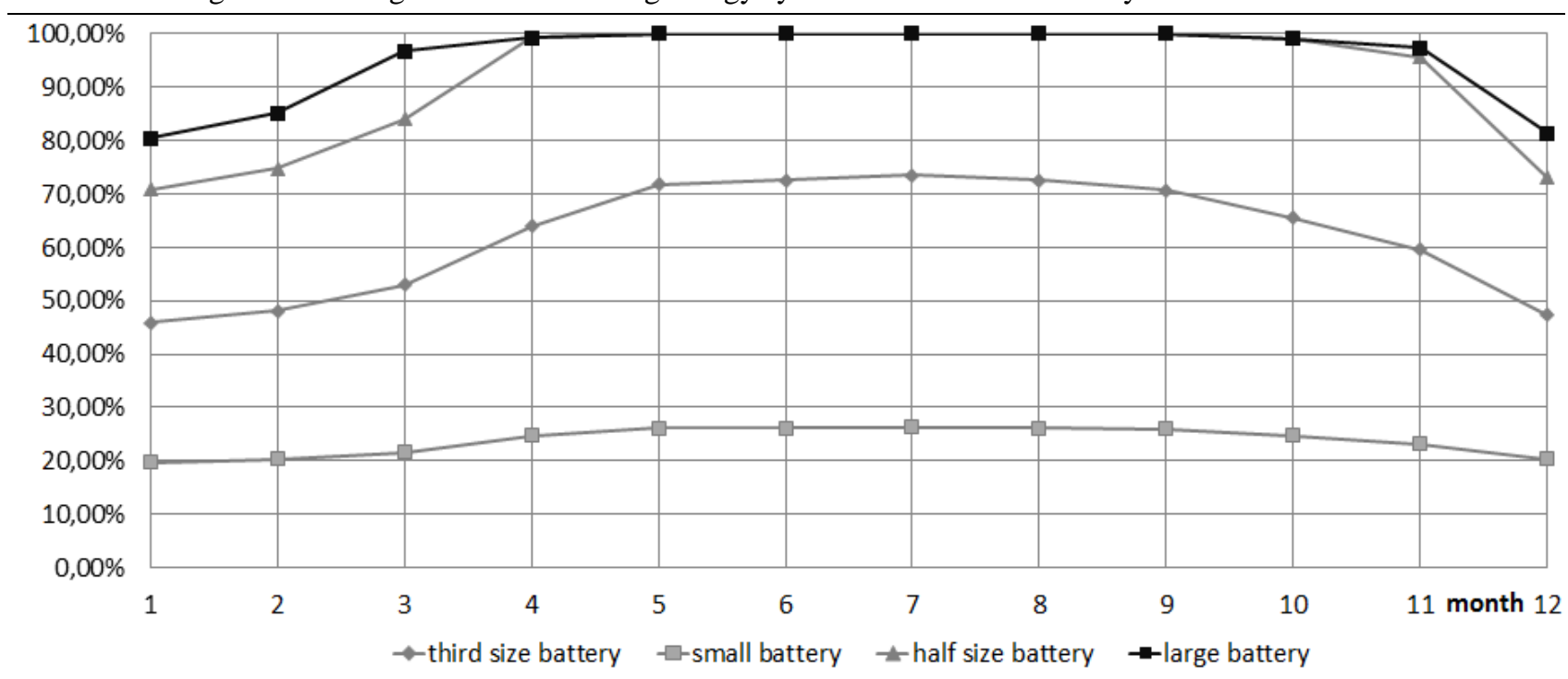

Fig. 9: Ratio of renewable energy to total vehicle energy demand

boiler).

The total $\mathrm{CO}_{2}$ emission of the whole energy system (building combined with vehicle) of the conventional system is about $55 \%$ higher than the one of the renewable system. This significant impact of the system on the environment can now be measured against investment cost or production resources.

The system can be optimized further to avoid grid storage (grid feed-in) or towards a maximum renewable vehicle mileage.

For the ratio between renewable energy and fossil fuels used to fulfill the individual mobility demand, i.e. the annual distance driven only using renewable energy, the size of the stationary battery is essential.

With the stationary battery, the energy income (CHP, PV at noon) can be decoupled from energy usage (charging at night).The basic simulated operational strategy (also defined in Modelica) was to charge the stationary battery whenever photovoltaic energy was available and to transfer this energy to the vehicle when docked. The difference to the energy requested by the vehicle was taken from CHP and grid. Values for losses in battery and converters matched those of the real components. This way a bigger amount of PV-energy in the stationary battery results in a higher renewable mileage.

To evaluate the influence of stationary battery capacity, the scenario was simulated with four sizes of relative $100 \%, 50 \%, 30 \%$ and $10 \%$. Fig. 9 shows that the PHEV with the biggest battery size is driven with an average ratio of $95 \%$ renewable energy during the year while the smaller ones have ratios of $91 \%, 62 \%$ and $24 \%$.

So, with a slightly worse primary energy balance (about 7\%) due to less grid-feeding, the biggest battery offers almost complete renewable mileage for the PHEV. With the half size battery, the coverage is still more than $90 \%$.

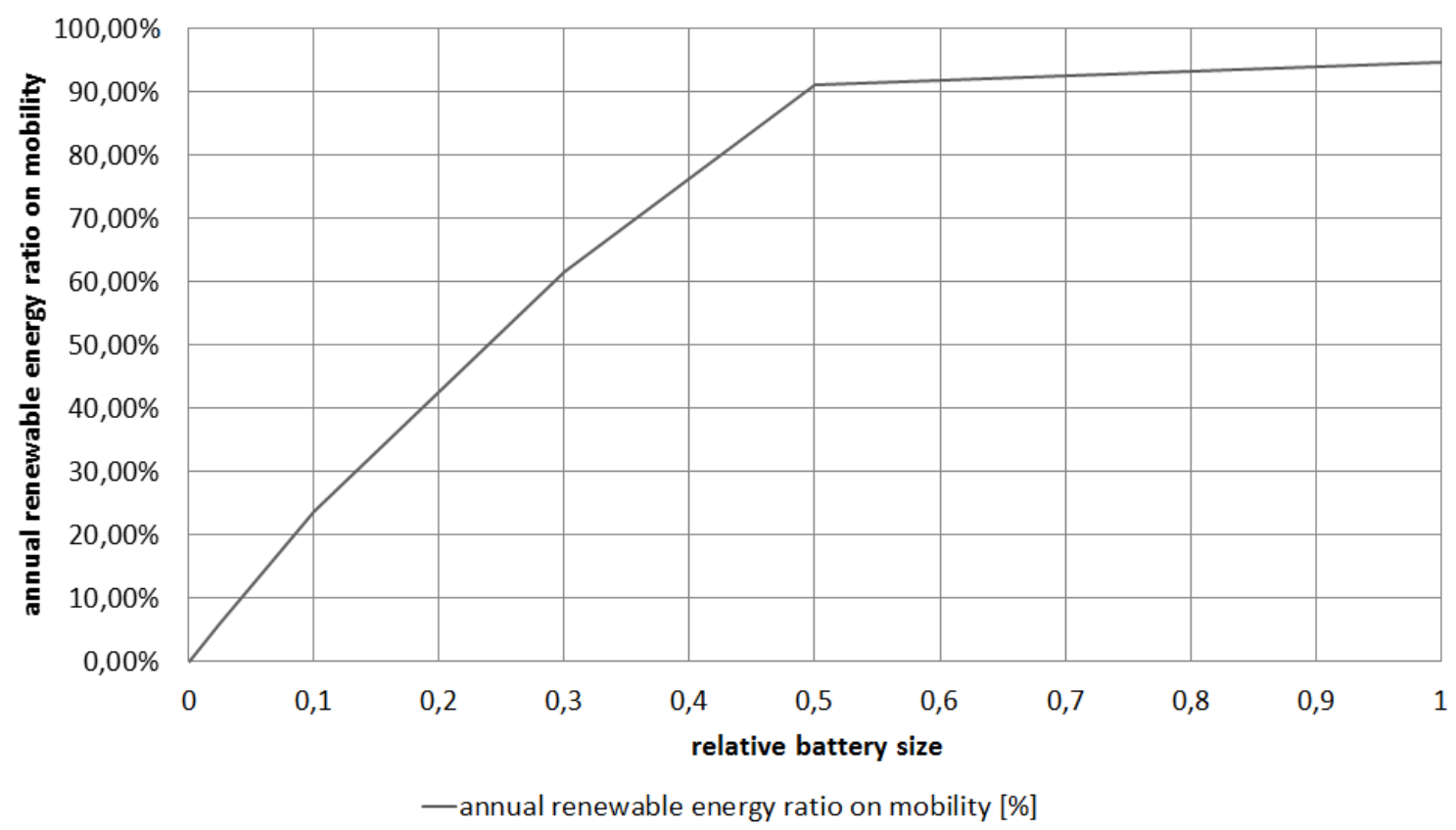

Fig. 10: annual renewable ratio of vehicle energy depending on relative battery capacity 


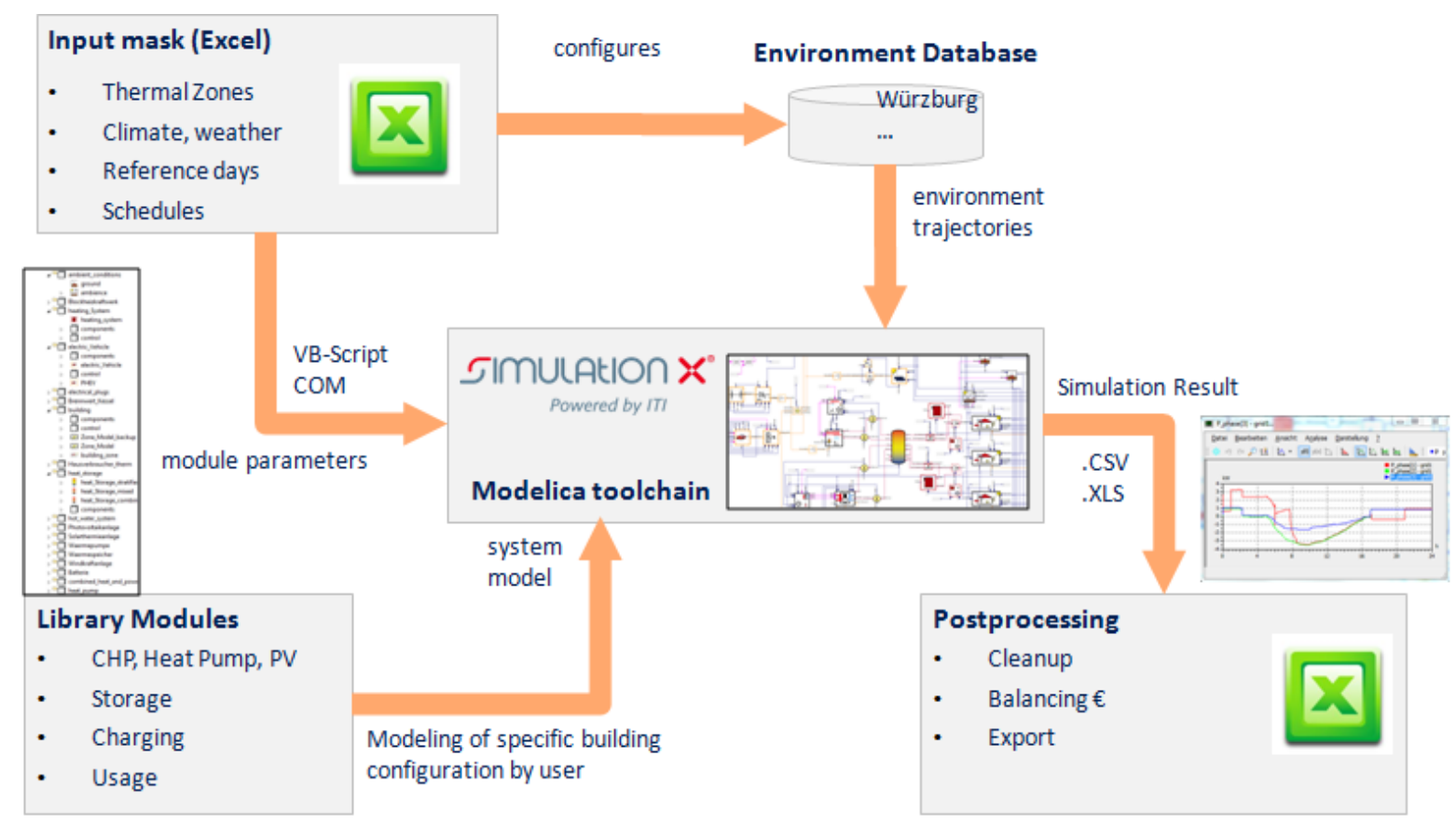

Fig. 11: Toolchain of simulation framework [1]

Obviously, with increasing battery size, i.e. capacity, the achievable annual renewable energy ratio on mobility increases monotonically (fig. 10). Because of the presented operating strategy for the photovoltaic system, the more capacity of the stationary battery is available the more renewably energy can be used to recharge the vehicle. An asymptotic maximum (fig. 10) occurs because the PHEV uses the internal combustion engine under cold outside temperatures, thus not using renewables.

Comparing battery costs leads to an optimum regarding battery size and annual renewable mobility energy ratio. This optimum can be calculated using annual capital costs for battery versus the achievable renewable energy ratio on annually driven distance.

The same evaluation and optimization could be done using different operational strategies, component (PV) sizes, vehicles or driving cycles. Even robustness to stochastic user behavior could be analyzed using the described holistic energy simulation approach.

\section{Summary and Conclusions}

The presented Modelica-based simulation library enables the modeling of various architectures for building energy systems including vehicles with (partly) electrified power trains. Simulation with these models creates a multitude of results, which can be used for evaluating and optimizing these systems using different criteria. Some criteria like battery size were presented within this paper. The more complex energy systems get, the larger the potential for optimization.

Besides the evaluation of energy system variants, the new Green Building framework (fig. 11) offers the capability of model-based development also for energy management algorithms in buildings or predictive renewable operation strategies for vehicles with electrified power trains.

Current work aims to improve the library with new component models like phase changing material (PCM) thermal storages and more top-level models for even easier use. A second major research objective is to create new energy management algorithms for the complete system using real-prediction and simulation [3].

Since renewable energies are still expensive in terms of money and production resources, an efficient use of these systems is essential.

\section{Acknowledgements}

The library and methods described are developed within the research project "Residence and Mobility". The research project is encouraged with subsidies from the European Union and the Sächsische Aufbaubank (SAB).

The simulation results presented in this paper arise from an exemplary research study, which was carried out in cooperation with Honda Research Institute Europe.

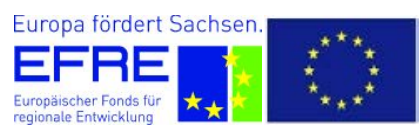




\section{References}

[1] Schwan, T., Unger, R., Baeker, B., Mikoleit, B., Kehrer, C.: Optimization of local renewable energy systems using automotive simulation approaches. $12^{\text {th }}$ Conference of International Building Performance Simulation Association, Sydney, 2011.

[2] Schwan, T., Unger, R., Baeker, B., Mikoleit, B., Kehrer, C.: Optimization-Tool for local renewable energy usage in the connected system: Building-eMobility. $8^{\text {th }}$ Modelica Conference. Dresden, 2011.

[3] Unger, R., Schwan, T., Mikoleit, B., Baeker, B.: "Residence and Mobility" - Renewable Energy Management in the linked system "Building - eMobility". $1^{\text {st }}$ International Energy Efficient Vehicle Conference. Dresden, 2011.

[4] Unger, R., Schwan, T., Mikoleit, B., Baeker, B.: Optimization-Tool for local renewable energy usage in the connected system: "Building-eMobility". 13 ${ }^{\text {th }}$ ITI Symposium. Dresden, 2010.

[5] Chrisofakis, E., Junghanns, A., Kehrer, C., Rink, A.: Simulation-based development of automotive control software with Modelica, $8^{\text {th }}$ Modelica Conference, Dresden, 2011.

[6] Fritzon, P.: Principles of Object-Oriented Modeling and Simulation with Modelica 2.1. Wiley-IEE Press, 2003.

[7] SimulationX-Hompage of ITI GmbH. http://www.simulationx.com

[8] Wetter, M., Zuo, W., Nouidui, T. S.: Recent Developments of the Modelica "Buildings" Library for Building Energy and Control Systems. $8^{\text {th }}$ Modelica Conference. Dresden. 2011.

[9] Kutter, S., Langhammer, S., Bäker, B.: eVehicleLib - Eine Modelica-Bibliothek zur Simulation von Fahrzeugen mit alternativen Antrieben; $20^{\text {th }}$ Symposium Simulationstechnik - ASIM 2009. Cottbus. 2009.

[10] Verordnung über energiesparenden Wärmeschutz und energiesparende Anlagentechnik bei Gebäuden - Energieeinsparverordnung, 2007.

[11] PVSol Hompage of The Solar Design Company.

http://www.solardesign.co.uk/index.php.
[12] TRNSYS-Homepage of Transsolar. http://www.transsolar.com/_software/docs/t rnsys/trnsys_uebersicht_de.htm 